\title{
PROTOTIPO DE CATÉTER ROBÓTICO CON SENSOR HÁPTICO BASADO EN VISIÓN
}

\author{
Almudena Bravo ${ }^{1}$, Ángel Rodríguez Castaño ${ }^{2}$, Blas M. Vinagre ${ }^{1}$, Inés Tejado ${ }^{1}$, \\ Enrique Mancha-Sánchez ${ }^{1}$, Paloma Rodríguez ${ }^{1}$, José Emilio Traver ${ }^{1}$ \\ ${ }^{1}$ Escuela de Ingenierías Industriales, Universidad de Extremadura, 06006 Badajoz \\ ${ }^{2}$ Escuela Técnica Superior de Ingeniería, Universidad de Sevilla, 41092 Sevilla \\ \{abravoi, bvinagre, itejbal, emancha, palomard, jetraverb\}@unex.es, castano@us.es
}

\section{Resumen}

El desarrollo de robots como asistentes de procedimientos quirúrgicos mínimamente invasivos está recibiendo un gran interés en los últimos años, fundamentalmente por ofrecer más precisión, flexibilidad y control respecto a las técnicas convencionales. En este artículo se presenta un primer prototipo de catéter autónomo basado en visión, realizando la navegación autónoma por medio del seguimiento de paredes inspirado en animales positivamente tigmotácticos. Además, se llevan a cabo distintos experimentos para verificar la funcionabilidad del prototipo.

Palabras clave: Cirugía mínimamente invasiva, catéter, navegación autónoma, sensor háptico basado en visión, nitinol.

\section{INTRODUCCIÓN}

La cirugía mínimamente invasiva (MIS por sus siglas en inglés) ha revolucionado las intervenciones quirúrgicas en las últimas dos décadas, utilizando procedimientos guiados por imagen para tratar y diagnosticar enfermedades. El uso de esta técnica está asociada a una reducción del dolor postoperatorio, pérdida de sangre, tasa de infección, tiempo de vuelta a las actividades normales, necesidades analgésicas postoperatorias y duración de estancia hospitalaria, en comparación con las intervenciones de cirugía convencionales $[2,7]$.

El campo de la robótica quirúrgica emplea desarrollos tecnológicos que incorporan sistemas de ingeniería para asistir en procedimientos médicos, y son especialmente útiles para MIS. Esta ayuda, que ha ganado una creciente aceptación en los últimos años, permite a los médicos llevar a cabo técnicas quirúrgicas con más precisión, flexibilidad y control de lo que es posible con las técnicas convencionales. Instrumentos quirúrgicos, tales como endoscopios o catéteres, entre otros, son dispositivos específicamente diseñados para ese fin. Estos instrumentos se introducen manualmente en el cuerpo, conducto o vaso, y se utilizan para el drenaje de fluidos, el tratamiento de enfermeda- des, o para llegar a sitios específicos del cuerpo para la administración de fármacos [6].

Los catéteres convencionales cuentan con un rango de movimiento y flexibilidad limitados, dependiendo en gran medida de la habilidad de los operadores, normalmente cirujanos, para maniobrar la punta del catéter a fin de lograr la interacción con el objetivo de una manera estable. Estar capacitado para desempeñar esta labor requiere mucho tiempo de aprendizaje y una costosa formación [10]. Además, la falta de información sobre la fuerza de contacto con el tejido, y la difícil anatomía de la vía, hace que este tipo de catéteres produzcan fallos de operación, debido al complejo funcionamiento en lo que a destreza y seguridad se refiere $[7]$.

Actualmente, las fuerzas se controlan mediante el tacto y, la localización del catéter, mediante fluoroscopia. Su uso aumenta a medida que las técnicas mínimamente invasivas se desarrollan para una gama cada vez más amplia de enfermedades. El guiado y la navegación de este tipo de catéteres provoca un aumento de la dosis de radiación en el paciente y personal intervencionista. Al ser unos procedimientos con necesidad de posicionamiento preciso, se hace extremadamente complicado solo con imágenes fluoroscópicas bidimensionales, por lo que al final son necesarias múltiples inyecciones de los agentes de contraste, adquisiciones de imágenes e intercambios de catéteres [11].

El desarrollo de catéteres de punta orientable (steereable catheter en inglés) permite paliar los inconvenientes de los catéteres convencionales. Se caracteriza por estar dotado de un extremo distal flexible y orientable, capaz de ser controlado remotamente. Se manipula mediante un mecanismo que puede ser accionado por operadores o por actuadores. Para que sea considerado catéter de punta orientable, el catéter debe cumplir una serie de requisitos detallados en [7], entre los que destacan: el uso de material biocompatible y esterilizable en su construcción, alto grado de destreza, intuitivo y de fácil manejo.

Las ventajas del catéter de punta orientable sobre el convencional son numerosas. La desviación 
del extremo distal es más controlable, mejora la accesibilidad en zonas de compleja anatomía, la estabilidad, además de reducir los tiempos de la fluoroscopia, permitiendo una disminución de la exposición a la radiación.

Entre este tipo de catéteres se encuentran los autónomos ( $\sin$ manejo por parte de un médico especializado). La navegación autónoma puede liberar al médico de la realización de tareas difíciles pero rutinarias, y poder centrarse en los componentes críticos de la misión. Para que el catéter pueda navegar con seguridad, es necesario conocer su ubicación y controlar las fuerzas que aplica al tejido [4].

Los animales positivamente tigmotácticos realizan un seguimiento de paredes, rastreando los límites de los contornos de los objetos, es una forma de explorar su entorno en ausencia de estímulos visuales [4].

Inspirados en la idea propuesta por Fagogenis et al. (2019) [4], este artículo presenta el diseño y desarrollo de un primer prototipo de catéter robótico con sensor háptico basado en visión. La navegación autónoma se realiza a través de algoritmos positivamente tigmotácticos, por medio de visión por computador, creando un contacto de baja fuerza con el tejido y permitiendo el seguimiento de paredes, gracias al sensor háptico y a la estructura diseñada para el posicionamiento del extremo, situada en la punta distal del catéter.

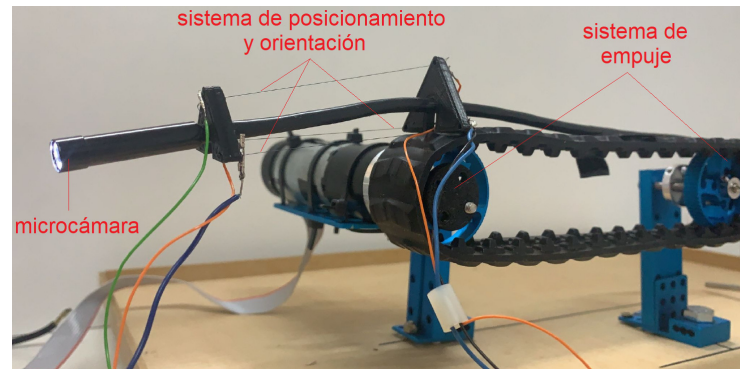

Figura 1: Prototipo de catéter autónomo (imagen real)

\section{DESCRIPCIÓN DEL PROTOTIPO}

En la Figura 1 se muestra el prototipo de robot catéter autónomo para navegación por medio de la visión háptica desarrollado. Está formado por tres sistemas dependientes entre sí ya que las acciones que efectúa uno son consecuencia de la actuación de otro:

- Sistema de posicionamiento de la punta del catéter, compuesto por tres "tendones" de ni- tinol, acoplados al extremo a través de dos piezas que hacen de columna vertebral, dotando de libertad de movimiento a la punta distal. Su actuación está gobernada por el sensor háptico basado en visión y el algoritmo diseñado.

- Sistema del sensor háptico basado en visión, que se encuentra en la punta distal del catéter, formado por una microcámara de endoscopia de $640 \times 480$ de resolución conectada por USB al ordenador que permite adquirir imágenes del entorno en tiempo real.

- Sistema de empuje, encargado del movimiento de avance (o retroceso, para la extracción) del catéter. Consta de un motor Maxon Motors de $24 \mathrm{~V}$, una controladora digital EPOS4 de Maxon Motors y un mecanismo de transmisión (correa más engranajes), todo anclado a una plataforma de madera para asegurar el sistema y evitar vibraciones innecesarias.

\subsection{PRINCIPIO DE FUNCIONAMIENTO GENERAL DEL PROTOTIPO}

El catéter navega mediante un sensor háptico basado en visión, un algoritmo que combina el seguimiento de paredes mediante contacto intermitente y el procesado de imágenes en tiempo real. La medida del contacto que ejerce el extremo del robot sobre una superficie se realiza de manera indirecta mediante visión, y más concretamente, segmentación de imágenes por umbralización del color.

En la Figura 2 se resume el funcionamiento general del catéter construido, se ha confeccionado un diagrama de flujo intuitivo para que se aprecie fácilmente la jerarquía de los movimientos y cómo son gobernados por el área del tejido observado.

La rutina comienza con la adquisición de la imagen, ésta se filtra para la eliminación del ruido (con un filtro Gaussiano 2D) y, después, se segmenta por umbralización en el espacio de color CIE $\mathrm{L}^{*} \mathrm{a}{ }^{*} \mathrm{~b}$ (que se refiere a luminosidad, coordenadas rojo-verde, coordenadas amarillo-azul, respectivamente), quedando la zona iluminada (es decir, la más cercana a la pared) resaltada después de haberse aplicado la máscara binaria.

Se realizan operaciones morfológicas de relleno de huecos y cierre, con elemento de estructuración en forma de disco de radio 3 . El cierre morfológico consiste en el encadenamiento de una dilatación seguida de una erosión, se utiliza para eliminar elementos salientes, conexiones finas o suavizar los contornos de un objeto. Como resultado, se obtiene una imagen binaria en la que la zona más 


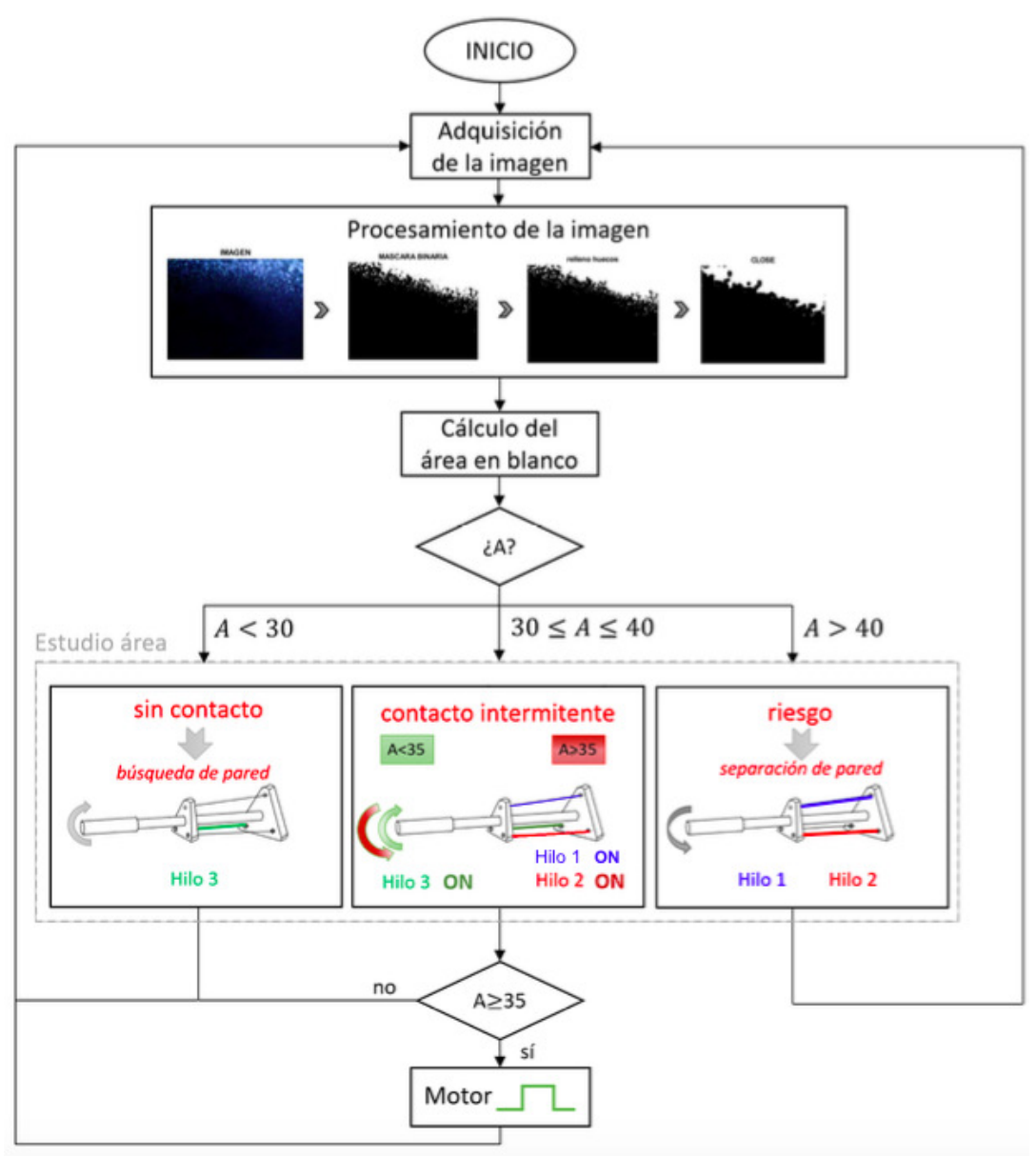

Figura 2: Diagrama general de la rutina de funcionamiento del prototipo

iluminada en la original, correspondiente al tejido próximo al extremo distal del catéter, queda en blanco (valor 1 en la imagen resultante), y el resto, en negro (valor 0). A partir de esta imagen, se calcula el área en blanco (se elige la más grande de todas las que puedan aparecer) respecto a la total de la imagen (en adelante, $A$ ), en porcentaje.

El área del tejido observado informa de cómo de cerca se encuentra el catéter de la pared, es decir, si bien se detecta área, no significa que se esté tocando el tejido. Para justificar la efectividad de este funcionamiento, se ha realizado una serie de instantáneas conforme el prototipo se aproxima la pared, quedando demostrado en la Figura 3, que no es hasta superar el rango del $35 \%$ aproximadamente cuando el catéter entra en contacto con la superficie a la que sigue.

Una vez el área del tejido próximo encerrado en el perímetro de la imagen está determinado, se realizan una serie de acciones dependiendo del porcentaje, provocando el movimiento hacia un lado u otro de la punta del catéter. En una zona concreta, denominada de seguimiento, será cuando el empuje por medio del motor se active e introduzca el catéter hacia el interior de la cavidad.

En la Figura 4 se incluye el diagrama de bloques del funcionamiento completo del prototipo, en la que el área de tejido visualizada hace una realimentación a sendos sistemas, el de posicionamiento de la punta, y el de empuje, arriba y abajo respectivamente, para que, dependiendo del porcentaje obtenido, se actúe en consecuencia.

El control del motor que produce el empuje se realiza mediante un control de dos grados de libertad (DOF), en el que en el interior del lazo de realimentación se incluye un control PID, y en lazo abierto, un filtro feedforward de ganancia unitaria.

\subsection{SISTEMA DE POSICIONAMIENTO DE LA PUNTA DEL CATÉTER}

Para que el extremo del catéter pueda tener maniobrabilidad para hacer el seguimiento de paredes y tener un alcance óptimo para las funciones en las que pueda ser requerido, es necesario dotar a la punta distal de un sistema que le permita 


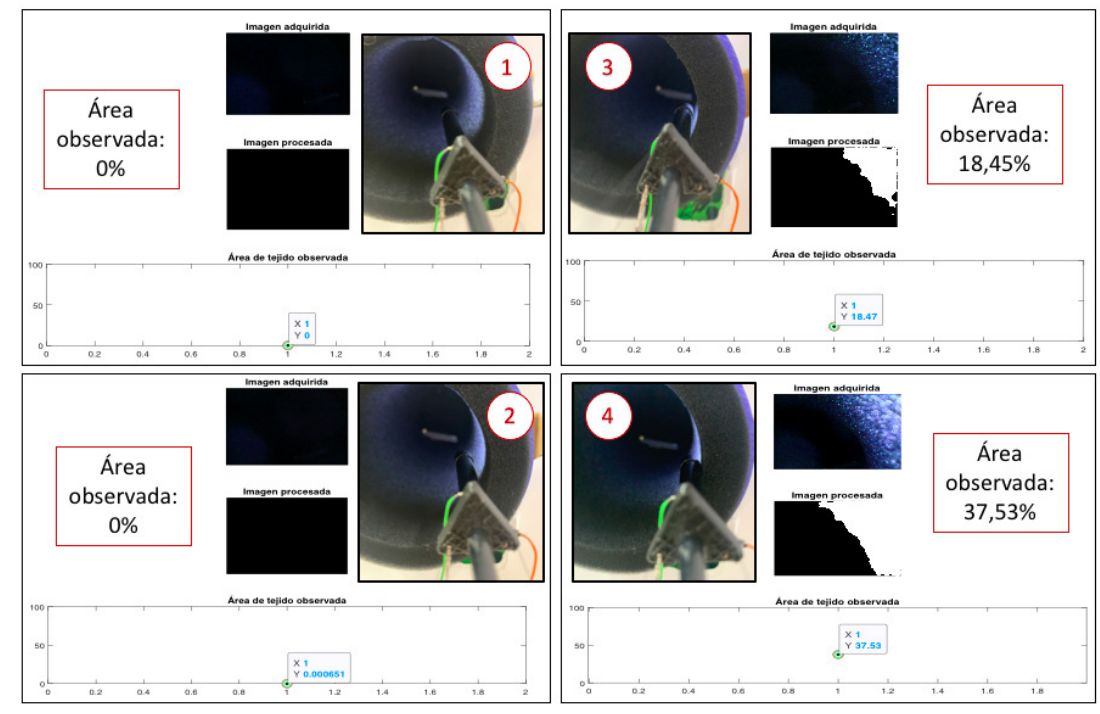

Figura 3: Justificación del rango de uso. Enumeradas en orden las instantáneas: En primer lugar alejado de la pared, detecta porcentaje de área cero; la segunda instantanea muestra el acercamiento del catéter a ésta pero con detección aproximada de cero; en la tercera detecta un porcentaje de área del 18,5\% pero se aprecia como no ha tocado aún la pared del tejido; la instantánea cuatro demuestra como en rangos superiores a $35 \%$ de área observado, el catéter está en contacto con el tejido.

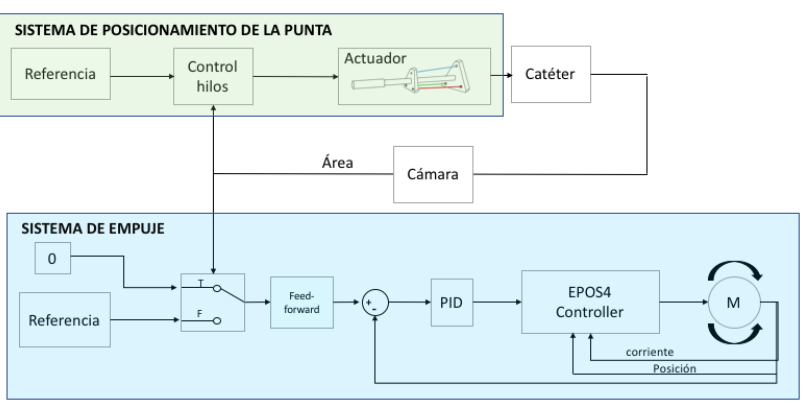

Figura 4: Diagrama de bloques del sistema completo

controlar su orientación. Para ello se coloca una estructura que lleva acoplado tres hilos de nitinol, una aleación (de niquel y titanio) de memoria de forma (SMA por sus siglas en inglés), equidistantes entre si $120^{\circ}$. Esto permitirá, gracias a la geometría de dichas piezas y a las propiedades del material super-elástico, nitinol, que el extremo se oriente hacia cualquier ángulo cuando se produzca un cambio en la estructura cristalina de alguno de los hilos, los cuales se contraen con el calor y vuelven a su posición inicial en su ausencia. Se aplicará corriente al hilo que se desee contraer mediante un circuito de excitación.

El nitinol se conecta entre la fuente de alimentación y las resistencias de drenaje del Mosfet. Cuando se aplica corriente a la puerta el circuito se cierra y el nitinol conduce, calentándose hasta contraerse más o menos rápido dependiendo de la tensión aplicada en la puerta. La fuente de tensión es de $5 V$, las resistencias de drenaje de $5 \Omega$ cada una y las de la puerta de $1,2 \mathrm{~K} \Omega$.

\subsubsection{Caracterización del movimiento del extremo distal}

Se puede calcular de manera aproximada cuánto desvía la estructura que se ha incorporado en el extremo la punta del catéter, por trigonometría. En la Figura 5 se ve el esquema simple de la desviación al igual que se denotan cada uno de los hilos. La Tabla 1 muestra los desplazamientos junto con los parámetros de los hilos de nitinol, siendo estos aproximadamente iguales se muestra una tabla genérica, donde $R_{\text {Marten }}$ es la resistencia del hilo cuando está en fase martensita; $R_{\text {Aust }}$ la resistencia del hilo cuando está en fase austenita; $L_{\text {Marten }}$ longitud del hilo cuando está en fase martensita, $L_{\text {Aust }}$ longitud del hilo cuando está en fase austenita y $\alpha$ el ángulo de desplazamiento.

Tabla 1: Características de la estructura

\begin{tabular}{|c|l|}
\hline Parámetro & Valor \\
\hline$R_{\text {Marten }}$ & $6,9 \Omega$ \\
$R_{\text {Aust }}$ & $7 \Omega$ \\
$L_{\text {Marten }}$ & $94 \mathrm{~mm}$ \\
$L_{\text {Aust }}$ & $92,6 \mathrm{~mm}$ \\
$\alpha$ & $9,9^{\circ}$ \\
\hline
\end{tabular}

En la Figura 6 se muestra el esquema de posicionamiento de la punta según los hilos que se encuentran escitados gracias al movimiento posibilitado por la estructura acoplada. 


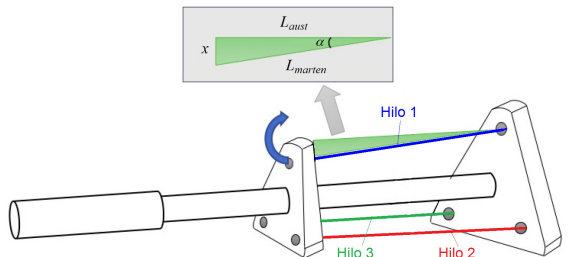

Figura 5: Cálculo de la desviación

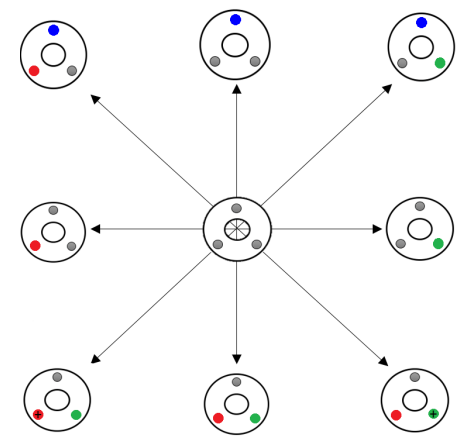

Figura 6: Esquema orientación de la punta dependiendo de hilos excitados (en color, los hilos excitados; el símbolo + representa una excitación de voltaje mayor).

\subsection{SENSOR HÁPTICO}

El principio de funcionamiento en el que se basa el sistema de navegación, explicado en el apartado 2.1, y que puede verse en la Figura 2, da como resultado un área, en porcentaje, que será la medida que controle en lazo cerrado el sistema. Las señales de referencia serán proporcionadas dependiendo del área, haciendo de nuevo incapié en que hasta no superar el rango del $35 \%$, no se considera que se esté tocando el tejido. También, añadir que el seguimiento de paredes está diseñado para seguir la pared derecha, y de acuerdo con el criterio propuesto en [4]:

- Si $30 \%<A$ entonces significa que el catéter se encuentra alejado de la zona de seguimiento, y por tanto, del tejido. Se excitará mediante el circuito diseñado el hilo 3 (en rojo en la Figura 2) para que el catéter vaya en busca del rango de seguimiento de paredes.

- Si $A>40 \%$, entra en zona de riesgo, considerándo que se ejerce más presión de la necesaria en el tejido. Se excitarán los dos hilos que se encuentran en el lado contrario al área detectada con máxima corriente, provocando la separación de la pared de la manera más rápida que ofrece el nitinol, es decir, en menos de un segundo.

- Si por el contrario, $30 \% \leq A \leq 40 \%$ se dice que está en zona del seguimiento de pared.
Este es el rango que se pretende mantener todo el tiempo. De manera que, cuando detecta que $30 \% \leq A<35 \%$, la excitación del hilo 3 (en verde en la Figura 2 ) se hará menor, es decir que la tensión que se aplica a la puerta del Mosfet que controla dicho hilo disminuye, haciendo que el hilo no se contraiga de una manera tan rápida porque podría pasarse de la zona de interés. Se busca que entre en el rango del 35-40\% de una manera lenta para que no se pase de los límites marcados y entre en zona de riesgo. Cuando $35 \% \leq A \leq$ $40 \%$ es el hilo 2 y el hilo 1 (marcado en rojo y azul, respectivamente, en la Figura 2) los que se excitan, mientras que se le da tensión nula a la puerta del hilo 3 , provocando que el catéter gire hacia la izquierda y se aleje de la pared. No se le dará máxima tensión a la puerta de ambos hilos para que no se vaya del rango que se considera de "seguimiento de pared". Es entonces cuando el ciclo vuelve a empezar.

En la parte superior de la Figura 6 se ilustra en lo que ocurre dentro del área de seguimiento del tejido.

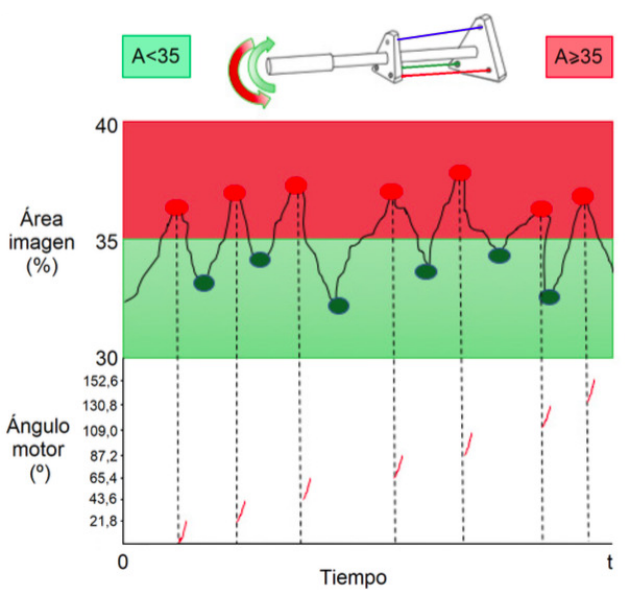

Figura 7: Esquema del modo de funcionamiento del seguimiento de paredes. Arriba el zigzageo del área a través de los rangos prefijados. Abajo, actuación del motor de empuje longitudinal para inserción del catéter

Los valores o rangos de excitación de los hilos en función del área se resumen en la Tabla 2; su ajuste se ha realizado de manera experimental. En los casos en los que especifica un rango, es decir, para el hilo 3 en las situaciones en las que $15 \leq A<30$ y $30 \leq A<35$, el voltaje de excitación se calcula de manera inversamente proporcional al área para el rango indicado. Cuando no se ha detectado área de tejido próxima, el voltaje de los hilos excitados es mayor. 


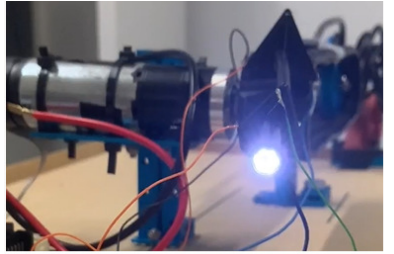

$t=0 \mathrm{~s}$

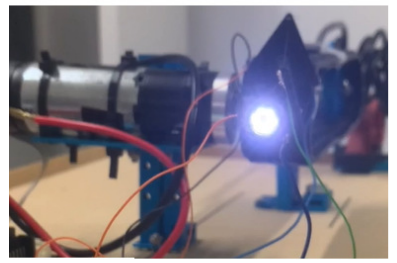

$t=10 \mathrm{~s}$

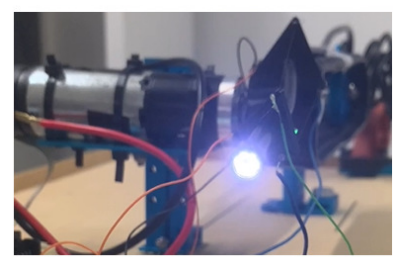

$t=2 \mathrm{~s}$

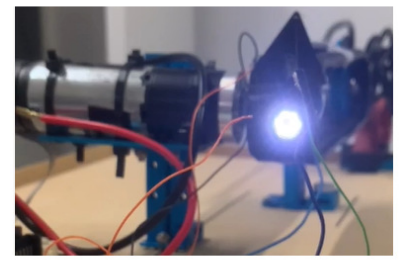

$t=12 \mathrm{~s}$

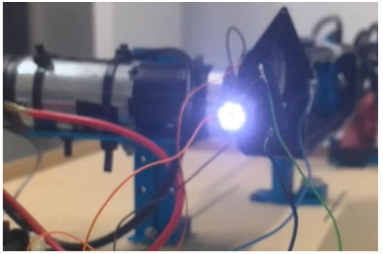

$t=4 \mathrm{~s}$

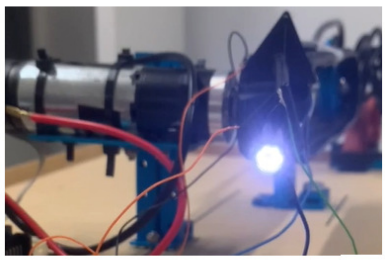

$t=14 \mathrm{~s}$

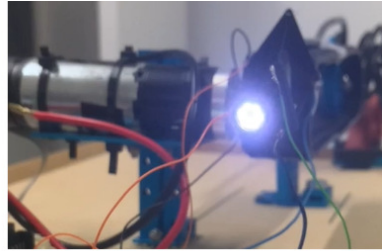

$t=6 \mathrm{~s}$

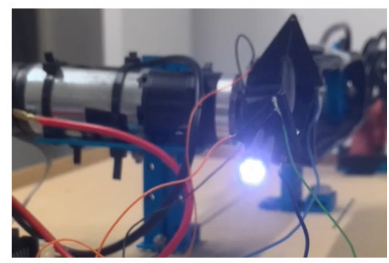

$t=16 \mathrm{~s}$

Figura 8: Experimento 1 del prototipo: fotogramas de los 16 primeros segundos del movimiento circular del extremo.

Tabla 2: Excitación de los hilos de nitinol según el área $A$.

\begin{tabular}{|c|c|c|}
\hline Área & Hilo & Rango voltaje (V) \\
\hline \hline $0<A<15$ & 3 & 3,3 \\
\hline $15 \leq A<30$ & 3 & $2,88-3,3$ \\
\hline $30 \leq A<35$ & 3 & $2,7-3,3$ \\
\hline $35 \leq A \leq 40$ & 1 & 1,94 \\
& 2 & 2,7 \\
\hline$A>40$ & 1 & 3,3 \\
& 2 & 3,3 \\
\hline
\end{tabular}

\subsection{SISTEMA DE EMPUJE LONGITUDINAL}

En un catéter autónomo surje la necesidad de tener un sistema que lo inyecte hacia el interior del paciente cuando sea necesario, como puede verse en $[9,3,4]$.

Tabla 3: Caracterización cinta transportadoracatéter

\begin{tabular}{|c|c|}
\hline Característica & Valor \\
\hline Giro $_{\text {Motor }}$ & $21,81^{\circ}$ \\
\hline Radio $_{\text {Rueda }}$ & $19,5 \mathrm{~mm}$ \\
\hline$L_{\text {circunferencia }}$ & $122,522 \mathrm{~mm}$ \\
\hline$L_{\text {Desplazada }}$ & $14,84355 \mathrm{~mm}$ \\
\hline
\end{tabular}

Una vez el catéter se encuentra en los rangos denominados de seguimiento de pared, este se acerca a la zona de área comprendida entre el 35-40\%, cuando la imagen procesada en el instante posterior a la llegada a este área es analizada, el sistema actúa en consecuencia excitando el hilo 2 en detrimento del hilo 1. Véase la Figura 7.

A la vez que el control de los hilos actúa sobre el hilo 1 y 2 para alejar el extremo de la pared, el conmutador del bloque del sistema de empuje (véase la Figura 4) da paso a la referencia de posición del motor, dando lugar al giro del motor. Puede comprobarse en la Tabla 3. Con el mecanismo de transmisión se consigue que este sistema empuje al catéter hacia el interior de la cavidad.

\section{EXPERIMENTOS}

Por el momento, se han realizado dos tipos de experimentos. El primero consiste en mover el catetéter de manera circular con el fin de demostrar la maniobrabilidad y capacidad que tiene para desarrollar su movimiento en los diferentes ángulos. El segundo experimento pretende verificar la capacidad de éste para realizar la navegación autónoma.

\subsection{EXPERIMENTO 1: MOVIMIENTO CIRCULAR DEL EXTREMO DEL CATÉTER}

El primer experimento llevado a cabo con el cáteter se corresponde con la verificación del correcto funcionamiento del sistema de posicionamiento y orientación del extremo del robot. En concreto, se desea realizar un movimiento circular. Para ello, las señales de excitación de cada uno de los hilos de nitinol se han definido como:

$$
v_{i}(t)=\frac{255}{2}+\frac{255}{2} \sin \left(\pi / 5 t+\phi_{i}\right)
$$

donde $\phi_{i}$ denota el desfase de la señal del hilo $i$, que vale $\pi / 2, \pi$ y 0 rad para los hilos 1,2 y 3 , respectivamente. Con esta excitación se persigue 
que, mientras un hilo comienza a enfriarse, los otro se calientan, y viceversa.

La Figura 8 muestra fotogramas de los 16 primeros segundos del movimiento obtenido con la excitación descrita, que es el tiempo que tarda en realizar un movimiento completo. Como se puede apreciar, éste se corresponde con un movimiento aproximadamente circular.

\subsection{EXPERIMENTO 2: NAVEGACIÓN HÁPTICA}

Se coloca el modelo de la Figura 1 en la entrada de un tubo de espuma negro, que se puede ver en la Figura 9. Se recuerda que está configurado para el seguimiento de la pared derecha, por lo que inicialmente si no detecta pared, se moverá hacia ese lado (hilo 3).

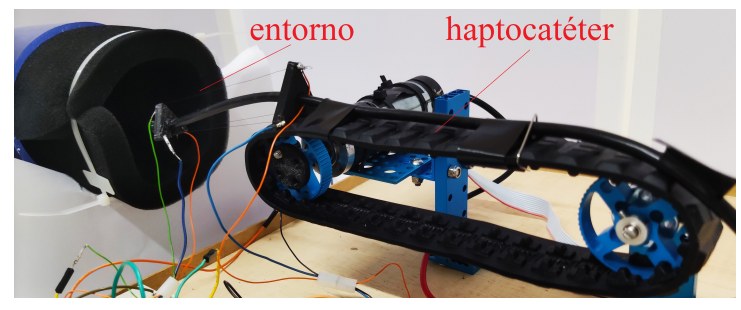

Figura 9: Configuración para el experimento.

La Figura 10 contiene los resultados obtenidos para dos de los experimentos llevados a cabo para la verificación de la navegación háptica. La Figura 10(a) muestra cómo cambia el área, mientras que la actuación del motor del sistema de empuje y las señales de activación de los hilos de nitinol se representan en las Figuras 10(b) y en las Figuras $10($ c) y $10(d)$, respectivamente. Estos resultados demuestran el correcto funcionamiento de este primer prototipo.

\section{CONCLUSIÓN}

Se presenta el sensor háptico basado en visión como una alternativa prometedora en el desarrollo de algoritmos de navegación autónoma basándose en animales positivamente tigmotácticos, que intentan mantener el contacto con sus alrededores, utilizando el seguimiento de las paredes en entornos desconocidos. Ya se desarrolló con éxito en [4] y en este trabajo se propone su desarrollo de una manera diferente pero con una base común. Además se muestran las posibilidades que brinda la aleación de memoria de forma nitinol a la hora de controlar y dotar de maniobrabilidad a un instrumento diseñado, en este caso, para intervenciones quirúrgicas, ya sean de inspección o diagnóstico, o para depositar un microrrobot en la zona de interés.

Se propone el uso de máscaras más robustas que permitan discriminar de una manera más efectiva el área del tejido cuando aún no se ha entrado en contacto, además de la inclusión de un control de fuerza por medio de un sensor que detecte la colisión en tiempo real, como ya hicieran $[1,5,8]$ entre otros, con celdas de carga, galgas extensiométricas y un sensor de desplazamiento de fibra óptica, respectivamente.

\section{Agradecimientos}

Este artículo ha sido financiado por la Consejería de Economía, Ciencia y Agenda Digital de la Junta de Extremadura mediante la Ayuda a Grupos GR18159, por la Agencia Estatal de Investigación mediante el proyecto PID2019-111278RB-C22 / AEI / 10.13039/501100011033, y por los Fondos Europeos de Desarrollo Regional "Una manera de hacer Europa".

\section{English summary}

\section{Prototype robotic catheter with vision-based haptic sensor}

\begin{abstract}
The development of robots as assistants for minimally invasive surgical procedures is receiving a great deal of interest in recent years, mainly because they offer more precision, flexibility and control than conventional techniques. This paper presents a first prototype of a vision-based autonomous catheter, performing autonomous navigation by means of wall-tracking inspired by positively tigmotactic animals. In addition, several experiments are carried out to verify the functionality of the prototype.
\end{abstract}

Keywords: Minimally invasive surgery, catheter, autonomous navigation, visionbased haptic sensor, nitinol.

\section{Referencias}

[1] Y. Asano, T. Kozuki, S. Ookubo, K. Kawasaki, T. Shirai, K. Kimura, K. Okada, and M. Inaba. A sensor-driver integrated muscle module with high-tension measurability and 


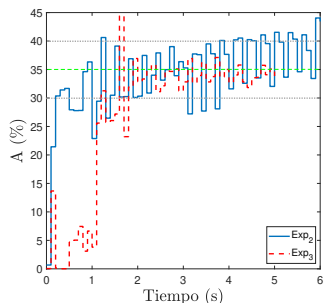

(a)

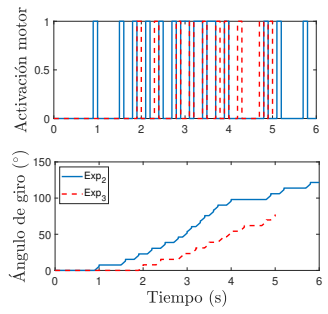

(b)

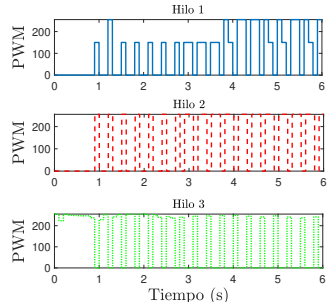

(c)

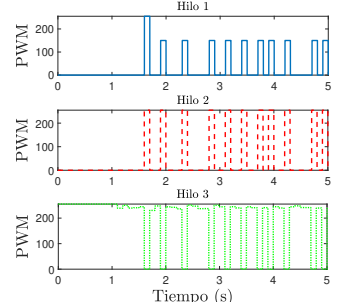

(d)

Figura 10: Experimentos finales con el haptocatéter relativos a la navegación háptica: (a) áreas (b) activación y ángulo del motor (c) excitación de los hilos (experimento 1) (d) excitación de los hilos (experimento $2)$.

flexibility for tendon-driven robots. In 2015 IEEE/RSJ International Conference on Intelligent Robots and Systems (IROS), pages 5960-5965. IEEE, 2015.

[2] M. A. Cooper, S. Hutfless, D. L. Segev, A. Ibrahim, H. Lyu, and M. A. Makary. Hospital level under-utilization of minimally invasive surgery in the united states: retrospective review. $B m j, 349,2014$.

[3] A. Degani, H. Choset, A. Wolf, T. Ota, and M. Zenati. Percutaneous intrapericardial interventions using a highly articulated robotic probe. In The First IEEE/RAS-EMBS International Conference on Biomedical Robotics and Biomechatronics, 2006. BioRob 2006., pages 7-12, 2006.

[4] G. Fagogenis, M. Mencattelli, Z. Machaidze, B. Rosa, K. Price, F. Wu, V. Weixler, M. Saeed, J. E. Mayer, and P. E. Dupont. Autonomous robotic intracardiac catheter navigation using haptic vision. Science robotics, 4(29), 2019.

[5] A. Garcia, V. Feliu, and J. Somolinos. Experimental testing of a gauge based collision detection mechanism for a new three-degreeof-freedom flexible robot. Journal of Robotic Systems, 20(6):271-284, 2003.

[6] C. Heunis, J. Sikorski, and S. Misra. Flexible instruments for endovascular interventions: Improved magnetic steering, actuation, and image-guided surgical instruments. IEEE Robotics 8 Automation Magazine, 25(3):71$82,2018$.

[7] X. Hu, A. Chen, Y. Luo, C. Zhang, and E. Zhang. Steerable catheters for minimally invasive surgery: a review and future directions. Computer Assisted Surgery, 23(1):2141, 2018.
[8] S. B. Kesner and R. D. Howe. Force control of flexible catheter robots for beating heart surgery. In 2011 IEEE international conference on robotics and automation, pages 1589-1594. IEEE, 2011.

[9] S. B. Kesner and R. D. Howe. Position control of motion compensation cardiac catheters. volume 27, pages 1045-1055. IEEE, 2011.

[10] E. D. Liapis, C. D. Avgerinos and A. Chatziioannou. The aortic arch: Markers, imaging, and procedure planning for carotid interven-tion. Vascular Disease Manage, 6(1), 2009.

[11] E. E. Sutton, B. Fuerst, R. Ghotbi, N. J. Cowan, and N. Navab. Biologically inspired catheter for endovascular sensing and navigation. Scientific reports, 10(1):1-10, 2020.

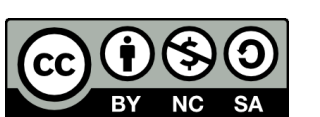

(C) 2021 by the authors. Submitted for possible open access publication under the terms and conditions of the Creative Commons Attribution CC BY-NC-SA 4.0 license (https://creativecommons.org/licenses/by-ncsa/4.0/deed.es). 